Check for updates

Cite this: RSC Adv., 2018, 8, 41389

Received 31st October 2018

Accepted 3rd December 2018

DOI: 10.1039/c8ra09005j

rsc.li/rsc-advances

\title{
Durable flame retardant polyacrylonitrile fabric via UV-induced grafting polymerization and surface chemical modification
}

\begin{abstract}
Yuanlin Ren, (D) ${ }^{* a b}$ Lina Jiang, ${ }^{a}$ Tian Tian, ${ }^{a}$ Xiaohui Liu ${ }^{* c}$ and Zhenbang Han ${ }^{a}$
To improve the flame retardancy of polyacrylonitrile (PAN) fabric, glycidyl methacrylate (GMA) was firstly grafted onto the surface of PAN fabric. Then, the GMA grafted PAN fabric (PAN-g-GMA) was chemically modified with hydrazine hydrate and phosphorus acid in sequence to obtain ammoniated PAN-g-GMA fabric (Am-PAN-g-GMA) and flame retardant PAN fabric (FR-PAN), respectively. The structures, thermal properties and combustion characteristics of the samples were researched in detail. The results indicate that the fire retardant PAN fabric has good char-forming ability. Cone calorimeter tests show that the total heat release (THR) of FR-PAN declines by 38.4\%, while the peak heat release rate (PHRR) of FR-PAN decreases by $60.2 \%$. Moreover, the total smoke production (TSP) and the peak smoke production rate (PSPR) of FR-PAN dropped from $1.5 \mathrm{~m}^{2}$ and $0.06 \mathrm{~m}^{2} \mathrm{~s}^{-1}$ for the control sample to $0.4 \mathrm{~m}^{2}$ and $0.01 \mathrm{~m}^{2}$ $\mathrm{s}^{-1}$, respectively, indicating excellent smoke repression performance. The LOI value of FR-PAN fabric was $29.3 \%$ after 30 washing cycles showing good washing resistance and excellent flame retardant durability.
\end{abstract}

\section{Introduction}

Polyacrylonitrile (PAN) fiber, one of the most important synthetic fibers, has been widely used for household and technical textiles because of its good warmth retention and resistance to light, mildew, etc. Unfortunately, PAN fiber is easy to ignite and burns intensely; what's more, toxic hydrogen cyanide will be released accompanied by smoke, which seriously restricts its wider applications. Therefore, development of a flame retardant PAN fiber is an alternative strategy to avoid this defect. It is interesting to find that phosphorus-containing species can impart acrylonitrile (AN) polymer, PAN fiber or its fabric good flame retardancy. In addition, PAN fiber ${ }^{1,2}$ and its fabric $^{3}$ will possess excellent flame retardancy when the phosphorus content in these materials is over $3 \mathrm{wt} \%$. Ammonium polyphosphate (APP), diammonium hydrogen phosphate and ammonium dihydrogen phosphate can be used alone or in combination as flame retardants to blend with PAN or AN polymers. In this blending method, these compounds can catalyze the cyclization of nitrile group $(\mathrm{CN})$, at the same time, the activation energy of cyclization decreases, as a result, the release of volatile products and smoke is restrained. ${ }^{4-6}$ In order

${ }^{a}$ School of Textiles, Tianjin Polytechnic University, 300387 Tianjin, China. E-mail: yuanlinr@163.com; Fax: +86-22-83958287; Tel: +86-22-83955353

${ }^{b}$ Key Laboratory of Advanced Textile Composite, Ministry of Education, Tianjin Polytechnic University, 300387 Tianjin, China

${ }^{c}$ School of Materials Science and Engineering, Tianjin Polytechnic University, 300387 Tianjin, China to increase the flame retardant durability of AN polymer, Wyman $^{7}$ copolymerized dialkyl- $p$-vinylbenzyl phosphonates with acrylonitrile and found that phosphorus acid decomposed from the copolymer could catalyze the copolymer's cyclization and char formation. Ren utilized $O, O$-diethyl-O-allylthiophosphate to copolymerise with acrylonitrile to increase the fire retardancy and the amount of char residue of the copolymer. ${ }^{8}$ However, the greater the steric effect of the comonomer, the more difficult the cyclization is of the copolymer. Therefore, some researchers attempted to take advantage of phosphorus free monomers, such as methacrylic acid and vinyl benzoic acid, ${ }^{9}$ methyl methacrylate ${ }^{10}$ and itaconic acid ${ }^{11-13}$ to copolymerize with acrylonitrile to promote the cyclization of the polymer owing to the catalysis of the carboxy group. Unfortunately, the flame retardancy of these copolymers without phosphorus content is far less than that of the phosphorus containing copolymers.

Ultraviolet (UV) grafting technique has been extensively applied for surface modification of materials. Compared with other grafting approaches, the UV-initiated photografting technique has many advantages, such as simple operation, low cost, especially little or no damage to the bulk properties of the materials. $^{\text {14-16 }}$ In the presence of photoinitiator, polymer macromolecular radicals will be formed when the polymer is exposed to UV irradiation. Then, the macromolecular radicals trigger the graft polymerization of monomers under UV irradiation. ${ }^{17}$ Liu prepared acrylamide-grafted nylon-6,6 fabric by UVinduced photografting technique and found that the fabric with the grafting percentage (GP) of $32.5 \mathrm{wt} \%$ had a limiting oxygen 
index (LOI) value of 26.2 and good washing durability. ${ }^{17}$ Similarly, Kundu synthesized a phosphorus and nitrogen-containing DOPO-based comonomer and made it graft onto nylon 6,6 fabric via UV grafting. The melt-dripping phenomenon was disappeared and the peak of heat release rate (PHRR) dropped by $22 \%{ }^{18}$ Furthermore, phosphorylated chitosan (PCS) was grafted onto the surface of the nylon 6,6 fabric through UVinduced photo-grafting technique followed by sol-gel coating. ${ }^{19}$ The results showed that the treated fabric had no melt dripping and the PHRR was decreased by $30 \%$. In our previous work, ${ }^{14}$ hydroxyethyl methacrylate (HEMA) was UV-induced to graft on the surface of PAN fabric. Then the grafted PAN fabric with 23 wt\% HEMA was phosphorylated to prepare flame retardant PAN fabric. The flame retardant PAN fabric had an LOI value of 32 and $40.5 \mathrm{wt} \%$ char residue at $800{ }^{\circ} \mathrm{C}$ in nitrogen. Tsafack $^{3}$ synthesized four phosphorus-containing monomers and utilized plasma-induced grafting technique to graft them on the surface of PAN fabric. The results showed when the phosphorus content on PAN fabric was $3.9 \mathrm{wt} \%$, the LOI value of flame retardant PAN fabric was 26.5.

Glycidyl methacrylate (GMA) is an epoxy group containing monomer capable of reacting with numerous groups such as carboxyl, $^{20}$ hydroxyl $^{21}$ and amine. ${ }^{22,23}$ As a result, many literatures have reported the successful grafting of GMA onto different substrates, for instance, PET fabric, ${ }^{24} \mathrm{PE}$ film ${ }^{25}$ water hyacinth fiber and cotton fabric. ${ }^{26}$

Herein, in order to improve the fire retardant performance of PAN fabric, GMA was grafted onto the surface of PAN fabric (PAN-g-GMA) by UV-induced grafting polymerization technique. Then, hydrazine hydrate and phosphoric acid were successively reacted with PAN- $g$-GMA to prepare flame retardant PAN fabric (FR-PAN). Furthermore, the structure, thermal properties and flame retardancy of the fabrics were all studied.

\section{Experimental section}

\subsection{Materials}

Polyacrylonitrile (PAN) fiber with 95 wt\% acrylonitrile and 5 wt $\%$ vinyl acetate was friendly supplied by Jilin Chemical Fiber Co., Jilin, China. Scoured plain-woven PAN fabrics (150 g $\mathrm{m}^{-2}$ ) were woven by School of Textiles, Tianjin Polytechnic University, Tianjin, China. Glycidyl methacrylate (GMA) was purchased from Nanjing Xiezun Chemical Reagent Technologies Co., Ltd, Nanjing, China. Benzophenone (BP) was obtained from Tianjin Guangfu fine Chemical Research Institute, Tianjin, China. Hydrazine hydrate, methanol, ethanol, acetone, urea and phosphoric acid were all purchased from Tianjin Fengchuan Chemical Reagent Technologies Co., Ltd., Tianjin, China. All the reagents were analytical grade and used without any further purification.

\subsection{Preparation of GMA-grafted PAN fabric (PAN- $g$-GMA)}

Polyacrylonitrile fiber fabric was cut into the appropriate size $(10 \mathrm{~cm} \times 10 \mathrm{~cm})$ and submerged in a solution containing specific concentration of BP, methanol and GMA for a period of time. Then, the fabric was taken out and put under a high pressure mercury lamp (500 W with a wavelength of $365 \mathrm{~nm}$ ) with $15 \mathrm{~cm}$ distance to irradiate for a certain period of time. Thirdly, the fabric was extracted with acetone in the soxhlet extractor for $12 \mathrm{~h}$ followed by repeatedly rinsing in distilled water to remove the excess BP, unreacted GMA and its homopolymer. Finally, the fabric was dried in an oven at $60{ }^{\circ} \mathrm{C}$ until a constant weight. The dried fabric was weighed on the analytical balance. The grafting percentage (GP) of GMA was calculated by the following equation:

$$
\operatorname{GP}(\%)=\left[\left(W_{2}-W_{1}\right) / W_{1}\right] \times 100 \%
$$

where, $W_{1}$ and $W_{2}$ are the weight of the fabric before and after grafting, respectively.

A series of fabrics with different grafting percentage (GP) were prepared. With the increase of GP, the physical properties of the fabrics decreased to some extent. Therefore, considering the efficiency of flame retardant modification and the physical properties of the fabric, the grafted fabric with a grafting percentage of $30 \mathrm{wt} \%$ was selected for the subsequent flame retardant modification.

\subsection{Preparation of flame retardant PAN fabric (FR-PAN)}

The PAN- $g$-GMA sample with $30 \mathrm{wt} \%$ of GMA was placed in a $250 \mathrm{~mL}$ three-neck round-bottom flask equipped with a stirrer. An aqueous solution of hydrazine hydrate was injected into the flask. The temperature of the solution was gradually heated to $90{ }^{\circ} \mathrm{C}$ for $2 \mathrm{~h}$ in a water bath. Then the fabric was washed three times with ethanol. After that the fabric was repeatedly washed with distilled water to remove the excess hydrazine hydrate, followed by drying in an oven for $12 \mathrm{~h}$. Thus, the aminated PAN$g$-GMA (Am-PAN- $g$-GMA) was obtained.

Next, Am-PAN- $g$-GMA sample was placed in a $250 \mathrm{~mL}$ threenecked round-bottom flask equipped with a stirrer, and the flask was placed in a water bath. An aqueous solution of phosphoric acid and urea was then injected into the flask. The temperature of the solution was gradually heated to $65{ }^{\circ} \mathrm{C}$ for $2 \mathrm{~h}$. Then the fabric was taken out and washed with distilled water many times to remove the excess reactants. Finally, the fabric was fully dried in an oven for $12 \mathrm{~h}$. Finally, the FR-PAN fabric was obtained with $44 \mathrm{wt} \%$ weight gain. The schematic route of preparing of FR-PAN is illustrated in Scheme 1.

\subsection{Characterization}

Attenuated total reflectance Fourier transform infrared spectroscopy (ATR-FTIR) was performed on a Bruker Vector 22 spectrometer. The spectra were acquired in the absorbance range of 4000-400 $\mathrm{cm}^{-1}$.

The X-ray photoelectron spectroscopy (XPS) spectra were recorded by a Thermofisher K-alpha photoelectron spectrometer using $\mathrm{Al} \mathrm{K} \alpha$ excitation radiation.

The thermogravimetric (TG) analysis was carried out by a thermogravimetric analyzer (Thermal Analysis Q600 SDT). All samples were heated from room temperature to $800{ }^{\circ} \mathrm{C}$ at a heating rate of $10{ }^{\circ} \mathrm{C} \mathrm{min}{ }^{-1}$ under air atmosphere. 
Step 1: Preparation of PAN-g-GMA

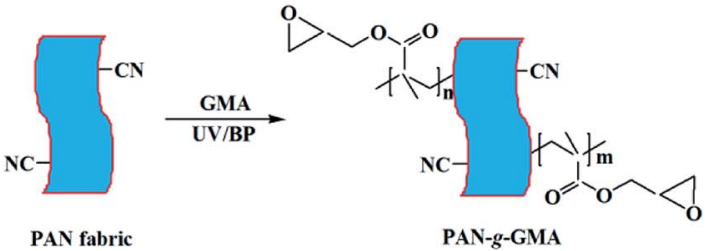

Step 2: Preparation of Am-PAN-g-GMA

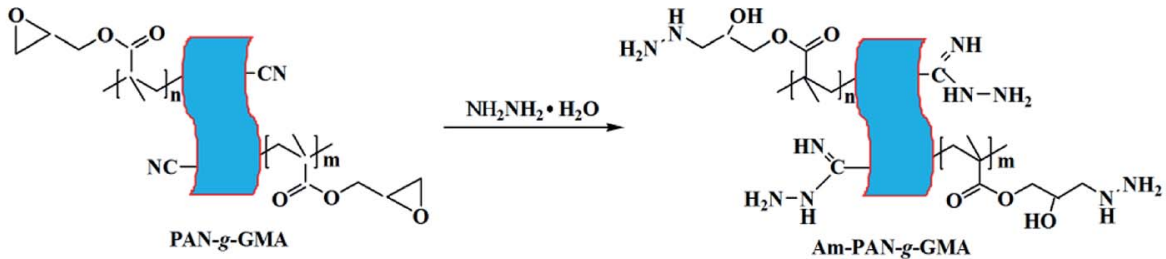

Step 3: Preparation of FR-PAN
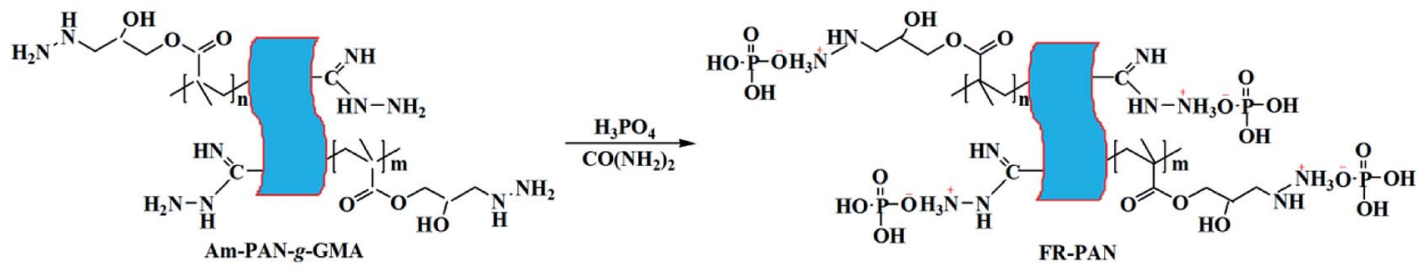

Scheme 1 The schematic of manufacturing flame retardant PAN fabric.

Differential scanning calorimeter (DSC) was carried out on a DSC200F3 (Netzsch, Germany). Each sample with about $5 \mathrm{mg}$ was sealed in aluminum sample pans. The samples were

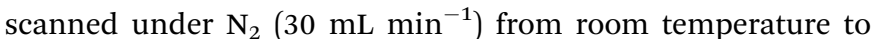
$350{ }^{\circ} \mathrm{C}$ at a heating rate of $10{ }^{\circ} \mathrm{C} \mathrm{min}^{-1}$. DSC curves of all the samples were obtained from a single heating process.

The surfaces of the samples were observed with a scanning electron microscope (SEM, Hitachi TM3030) to analysis the changes in surface morphology.

The combustion performance of the samples with the size of $100 \mathrm{~mm} \times 100 \mathrm{~mm} \times 2 \mathrm{~mm}$ was accessed by a cone calorimeter (FTT, UK) according to ISO 5660-1 under an irradiative heat flux of $35 \mathrm{~kW} \mathrm{~m}{ }^{-2}$ in horizontal configuration. The time to ignite (TTI), heat release rate (HRR) and the peak of HRR (PHRR), total heat release (THR), total smoke production (TSP), smoke production rate (SPR) and peak of SPR (PSPR) were all evaluated.

The flammability of the samples was measured by limiting oxygen index (LOI) according to GB 5454-1997 on a HC-2 limited oxygen index instrument. The samples with the size of $58 \mathrm{~mm}$ $\times 150 \mathrm{~mm}$ were ignited several seconds in methane flame and tested for five times.

In order to verify the flame retardant durability of the fire retardant PAN fabric, the fabric with the size of $5 \mathrm{~cm} \times 10 \mathrm{~cm}$ was washed according to AATCC Test Method 61-2003 test no. $1 \mathrm{~A}$ with $0.37 \mathrm{wt} \%$ detergent. One washing cycle lasting for 45 min equals to five commercial launderings. Accordingly, the LOI values of the fabrics after different washing cycles were test.

\section{Results and discussion}

\section{$3.1 \quad$ FTIR analysis}

Fig. 1 shows the FTIR spectra of the pristine fabric, PAN- $g$-GMA, and FR-PAN samples. For the original PAN fabric, the peak located at $2242 \mathrm{~cm}^{-1}$ is due to the stretching vibrations of the typical functional group of $-\mathrm{CN}$. The peaks appeared at $2934 \mathrm{~cm}^{-1}$ and $1446 \mathrm{~cm}^{-1}$ attributes to the stretching and bending vibrations of the $\mathrm{C}-\mathrm{H}$ bond in $-\mathrm{CH}_{2} \cdot{ }^{27}$ Besides, a peak

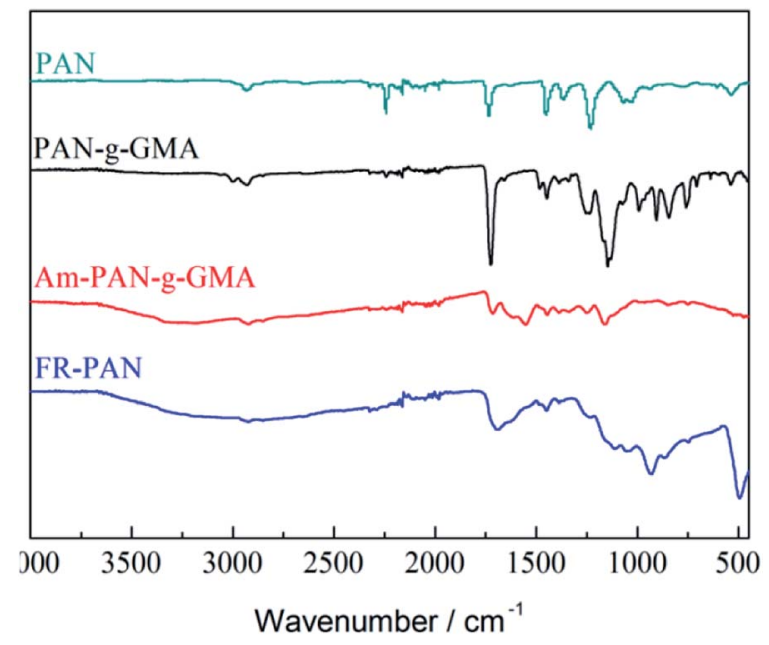

Fig. 1 FTIR spectra of PAN and modified PAN fabrics. 


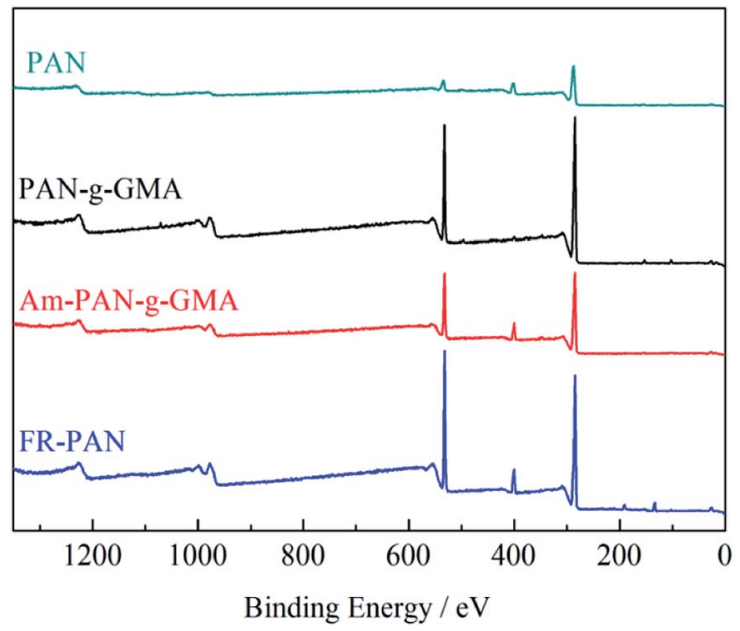

Fig. 2 XPS spectra of PAN and modified PAN fabrics.

appeared at $1732 \mathrm{~cm}^{-1}$ belongs to the stretching vibrations of $\mathrm{C}=\mathrm{O}$ in the carboxyl group, which is also the typical functional group of acrylonitrile-vinyl acetate copolymer fiber fabric used in our previous work. ${ }^{28}$

For the PAN- $g$-GMA fabric, the adsorption peak at $1732 \mathrm{~cm}^{-1}$ is significantly enhanced attributing to the $\mathrm{C}=\mathrm{O}$ group of the grafted GMA. Meanwhile, the appeared three bands at $990 \mathrm{~cm}^{-1}, 914-854 \mathrm{~cm}^{-1}$ and $764 \mathrm{~cm}^{-1}$ are the characteristic peaks of the epoxy group in GMA. ${ }^{29}$ The results demonstrate that GMA has been successfully grafted onto the surface of the PAN fabric.

As far as the spectrum of Am-PAN- $g$-GMA is concerned, the typical bands of the epoxy group disappear. Alternatively, a wide band appears at $3700-2984 \mathrm{~cm}^{-1}$ belonging to the $-\mathrm{OH}$ and $-\mathrm{NH}_{2}$ resulting from the reaction of epoxy groups with hydrazine hydrate. ${ }^{8}$ In case of FR-PAN, the band located at $1186 \mathrm{~cm}^{-1}$ is caused by $\mathrm{P}=\mathrm{O}$ and $\mathrm{P}-\mathrm{O}$ group, while the peak at $929 \mathrm{~cm}^{-1}$ attributes to the $\mathrm{O}-\mathrm{P}-\mathrm{O}$ group. These results confirm the amination and phosphorylation of the PAN-g-GMA fabric, in other words, flame retardant PAN fabric has been successfully prepared.

\subsection{XPS analysis}

The elemental components on the surface of the fabrics are analyzed by XPS, as shown in Fig. 2 and Table 1. For the original PAN fabric, three typical absorption peaks at 532, 405 and $286 \mathrm{eV}$ are due to O1s, N1s and C1s, respectively. ${ }^{14}$ For the PAN$g$-GMA fabric, it is clearly that the peaks of O1s and N1s increase greatly, resulting from the grafted GMA. In case of Am-PAN-gGMA, the peak of N1s increases. For the FR-PAN fabric, a new absorption peak owing to $\mathrm{P} 2 \mathrm{p}$ appears at $140 \mathrm{eV}$ while the peak of O1s increases obviously. ${ }^{30}$ These results again prove the successful modification of the PAN fabric, which are agreed with the results of the FTIR analysis.

\subsection{Thermal analysis}

The thermal properties of the pristine fabric, PAN- $g$-GMA, AmPAN- $g$-GMA and FR-PAN are assessed by TG-DTG in air
Table 1 Chemical composition of the different fabrics

\begin{tabular}{lllll}
\hline Sample & C1s (\%) & O1s (\%) & N1s (\%) & P2p (\%) \\
\hline PAN & 81.93 & 10.86 & 7.21 & 0 \\
PAN- $g$-GMA (30 wt\%) & 73.46 & 22.43 & 1.15 & 0 \\
Am-PAN-g-GMA & 68.99 & 21.76 & 7.69 & 0 \\
FR-PAN & 64.14 & 25.81 & 7.18 & 2.86
\end{tabular}

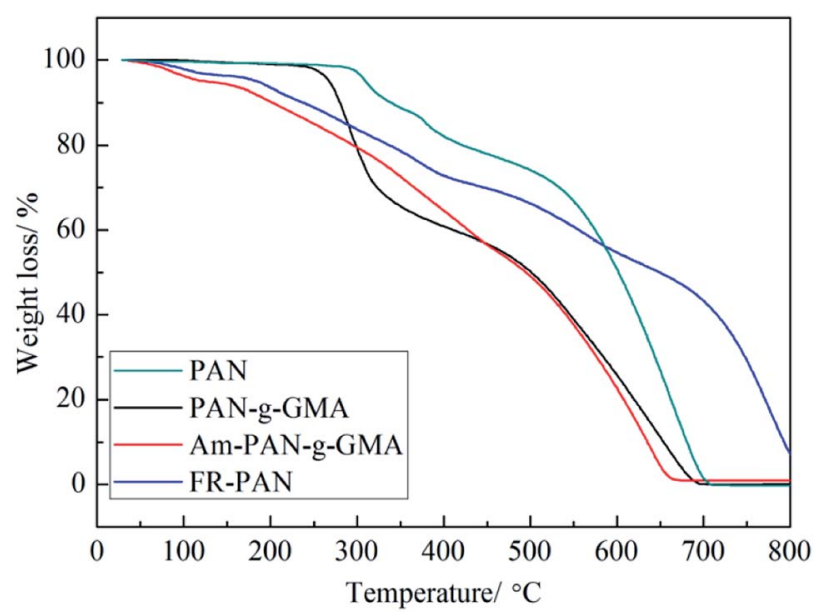

Fig. 3 TG curves of PAN and modified PAN fabrics.

atmosphere. The TG-DTG curves of each sample are shown in Fig. 3 and 4.

For the original PAN fabric, three weight loss steps are observed attributing to the cyclization, decompositioncarbonization and thermo-oxidation of char, ${ }^{31-33}$ as can be seen in Fig. 3. The first stage occurs at about $288{ }^{\circ} \mathrm{C}$ and the peak temperature of the first weight loss is at $308^{\circ} \mathrm{C}$. In this stage, the weight loss attributes not only to the oligomerization reaction between the adjacent cyano, but also to the released gases, for example, ammonia and nitriles resulting from the decomposition of the PAN side group. ${ }^{34}$ The second stage begins at $366^{\circ} \mathrm{C}$. In this region, the decomposition-carbonization process of the cyclic structure occurs and produces a certain amounts of gases, such as ammonia, hydrogen and hydrogen cyanide. As a result, a graphic carbon structure is formed in this period..$^{31}$ The third step occurs at above $480{ }^{\circ} \mathrm{C}$ owing to the thermal oxidation decomposition of the char residue produced in the first two stages.

For PAN-g-GMA, two main weight loss steps appeared. The peak temperature of the first mass loss is lower than that of the control sample attributing to the earlier degradation of GMA. The second mass loss is wider than the first one and is similar to that of the original PAN.

As far as Am-PAN-g-GMA is concerned, the weight loss is not clearly observed in TG curves, however, as seen from DTG curves, the weight loss is relatively complex, and can be mainly divided into five stages. The first stage located at $90{ }^{\circ} \mathrm{C}$ corresponding to the release of the adsorbed water due to the moisture adsorption ability of the amino groups in Am-PAN- $g$ GMA. The second weight loss is at $188{ }^{\circ} \mathrm{C}$ owing to the release of 


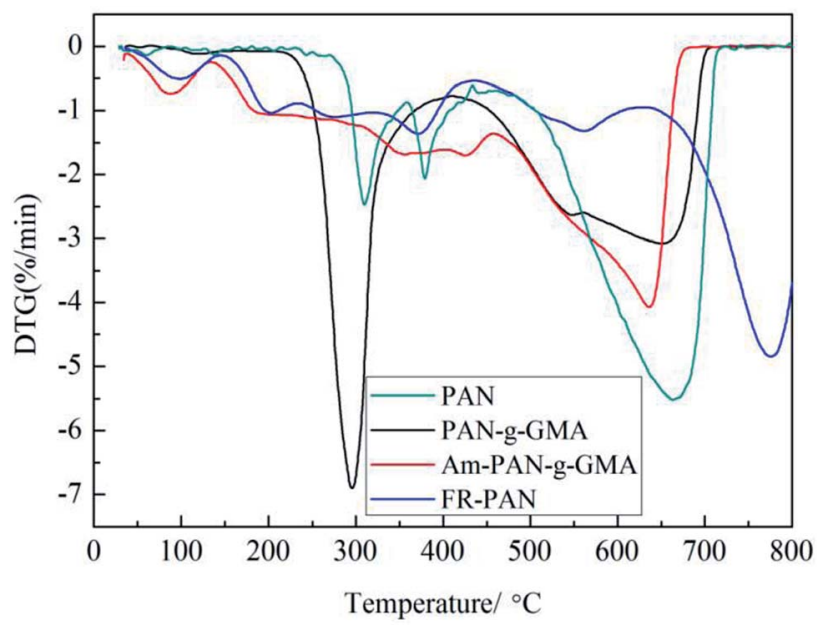

Fig. 4 DTG curves of PAN and modified PAN fabrics.

water and ammonia decomposed from the nitrogen-, hydroxycontaining groups. The third and the fourth weight losses are due to the decomposition-carbonization of the cyclic structure, which is similar to that of the control sample. Similarly, the last weight loss attributes to the thermal oxidation decomposition of the char residue.

In case of FR-PAN sample, the weight loss is more complex, and six weight losses are appeared. The first one located at $95{ }^{\circ} \mathrm{C}$ is ascribed to the release of the absorbed water, which is due to the moisture adsorption of amino groups and phosphoric hydroxyl groups. The second one lying at $202^{\circ} \mathrm{C}$ attributes to the released water and the decomposition of the grafted pendent groups. The third and the fourth weight losses are due to the further decomposition of the grafted pendent groups and the dehydrogenation of cyanide cyclization. The fifth one is the decomposition-carbonization process of the formed cyclic structure and the last one is the thermal oxidation decomposition of the char residue produced in previous stages.

\subsection{DSC analysis}

Fig. 5 shows the DSC curves of PAN, PAN-g-GMA, Am-PAN-gGMA and FR-PAN samples. The DSC curves are carried out under nitrogen atmosphere, therefore, oxidative reactions do not occur during the testing procedure. DSC curves of all the samples are acquired from ambient temperature to $350{ }^{\circ} \mathrm{C}$, so the exotherms peaks of all the samples are attributed to the cyclization reactions, which are initiated through a free radical mechanism. ${ }^{35}$ As shown in Fig. 5, there is only one sharp and shoulder-containing exothermic peak located at $305^{\circ} \mathrm{C}$ in PAN fabric with $-514 \mathrm{~J} \mathrm{~g}^{-1}$ heat release, which is caused by the breakage of the molecular chains and weight loss. In addition, it is agreed with the results of TG analysis.

In case of PAN-g-GMA, there is also one exothermic peak, and the temperature of the sharper peak lied at $311^{\circ} \mathrm{C}$, which is a little higher than that of the control fabric. This may be due to the fact that the grafted GMA groups weakens the interaction between nitrile groups and restrains the cyclization reaction,

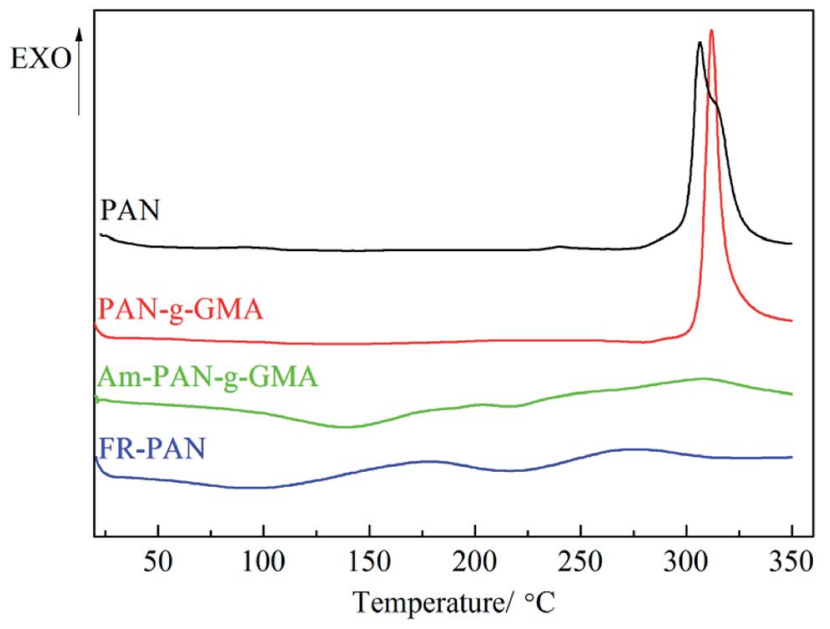

Fig. 5 DSC curves of PAN and modified PAN fabrics.

resulting in the cyclization temperature increasing. The heat release $\left(-363 \mathrm{~J} \mathrm{~g}^{-1}\right)$ is quick and concentrated and is lower than that of the pristine fabric $\left(-514 \mathrm{~J} \mathrm{~g}^{-1}\right)$.

For Am-PAN- $g$-GMA, two wider peaks appeared, the endothermic peak $\left(133 \mathrm{~J} \mathrm{~g}^{-1}\right)$ locating at $140{ }^{\circ} \mathrm{C}$ attributes to the release of adsorbed water. The exothermic peak $\left(-175 \mathrm{~J} \mathrm{~g}^{-1}\right)$ lying at $307^{\circ} \mathrm{C}$ is assigned to the cyclization reactions initiated by the grafting pendent groups through an ionic mechanism, which broadens the exothermic peak and avoids centralized heat release.

In case of FR-PAN, there are two exothermic peaks and the cyclization reactions can be initiated through both radical and ionic mechanism..$^{36}$ The lower exothermic peak is due to the cyclization reactions, which is much lower than that of the control sample, PAN- $g$-GMA and Am-PAN- $g$-GMA, indicating that the cyclization reactions are easier to be initiated in FR-PAN than in the control, PAN- $g$-GMA and Am-PAN- $g$-GMA samples. In addition, the higher exothermic peak of FR-PAN is also lower than that of other samples. The heat release of the two exothermic peaks is -49 and $-100 \mathrm{~J} \mathrm{~g}^{-1}$, respectively. It is reported that single exothermic peak in DSC curve is not conducive to improving the stabilization of PAN. ${ }^{34}$ Therefore, two exothermic peaks appeared in FR-PAN indicates that FR-PAN can efficiently promote the cyclization reaction and improve the stabilization of PAN than the control, PAN- $g$-GMA and AmPAN- $g$-GMA samples, as is proved by TG analysis.

\subsection{Combustion properties}

Cone calorimetric (CC) tests were performed in order to analyze the combustion of the FR-PAN fabric by simulating the fire environment in the real fire. The heat release rate (HRR), total heat release (THR), smoke production rate (SPR) and total smoke production (TSP) of the original PAN and FR-PAN fabrics are all obtained and shown in Fig. 6. In addition, the corresponding data including the time to ignite (TTI), peak of HRR (PHRR), peak of SPR (PSPR), average mass loss rate (aMLR) and the residue mass are summarized in Table 2. 

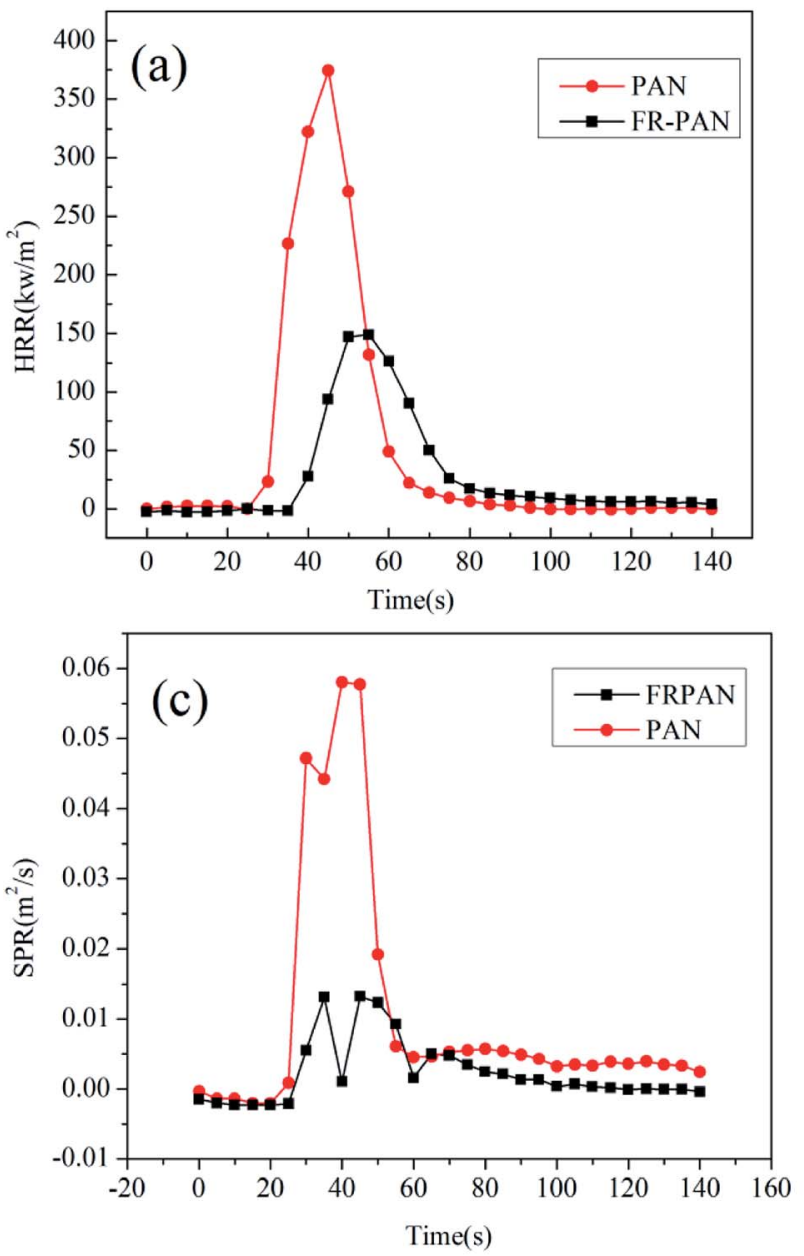
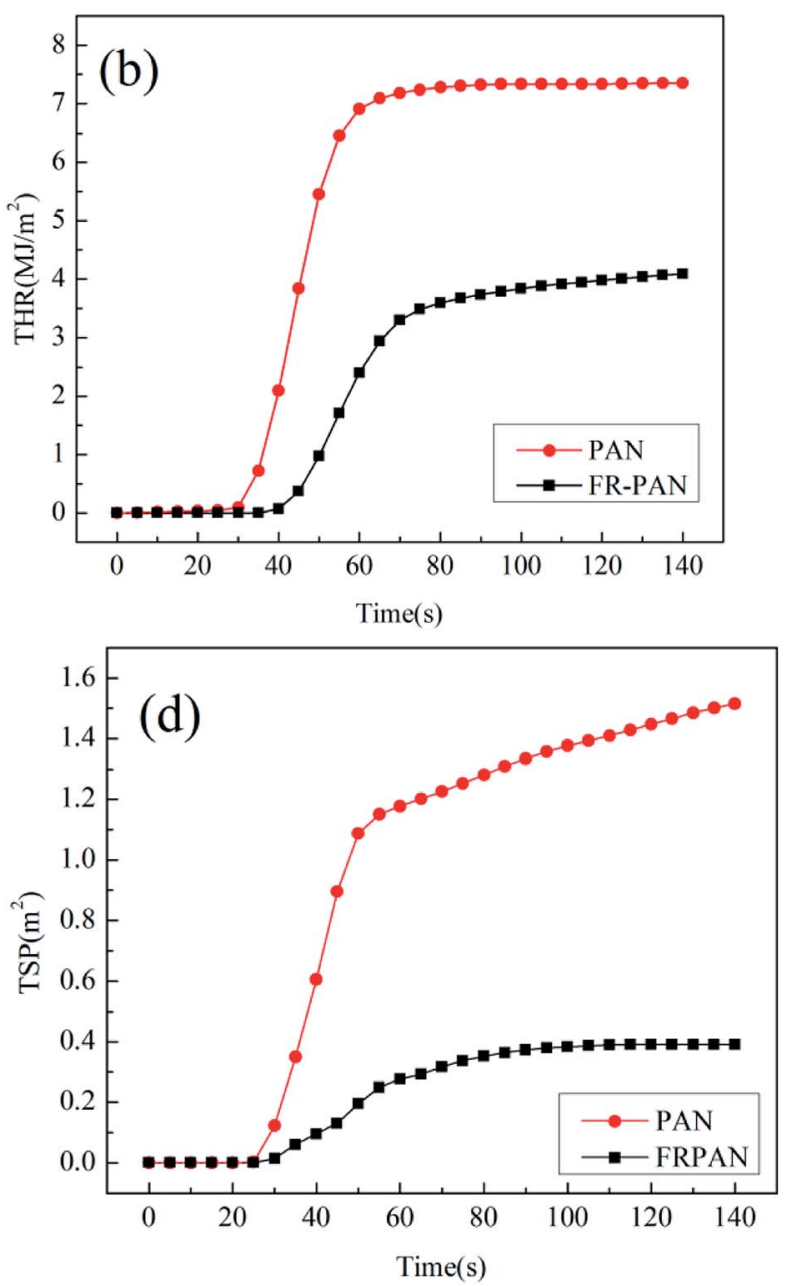

Fig. 6 HRR (a), THR (b), SPR (c) and TSP (d) curves of PAN and FR-PAN fabric.

As shown in Table 2, the TTI value increases from $25 \mathrm{~s}$ of the control PAN fabric to $33 \mathrm{~s}$ of the FR-PAN fabric. As TG explained above, FR-PAN will undergo thermal decomposition at relative lower temperature to release the bound water. The released water will take away part of the heat, in other words, in order to ignite the sample, more heat and time are needed. Therefore, TTI of FR-PAN is higher than that of the control sample. The aMLR of the FR-PAN fabric is reduced a half compared with that of the control PAN fabric, while the residual mass of the FR-PAN fabric after combustion increases obviously, which confirms the results of TG test. Besides, the fire growth rate index (FIGRA) calculated by the ratio of PHRR and time to PHRR, decreases significantly. In general, a lower FIGRA implies that the time to deflagration is delayed, which makes people in the scene of a fire have enough time to escape and fire extinguishers arrive. ${ }^{37}$ These results demonstrate the suppression for the fire occurrence and the fire spread of the FR-PAN fabric, ${ }^{38}$ which can significantly extend the time to escape in a real fire.

Heat release properties are of great significance in assessing the flame retardant properties of materias. As can be seen in Fig. 6(a) and (b), compared with the original PAN fabric, the FRPAN fabric exhibits a decrease of PHRR and THR. The PHRR decreases from $374.4 \mathrm{~kW} \mathrm{~m}^{-2}$ to $149.0 \mathrm{~kW} \mathrm{~m}^{-2}$, while the time to PHRR extends from $45 \mathrm{~s}$ to $55 \mathrm{~s}$. At the same time, the THR decreases from 7.3 $\mathrm{MJ} \mathrm{m}^{-2}$ to $4.1 \mathrm{MJ} \mathrm{m}^{-2}$. These results show the flame retardant properties of the FR-PAN fabric are highly improved.

The SPR and TSP curves of the original PAN fabric and the FR-PAN fabric are respectively shown in Fig. 6(c) and (d). For the FR-PAN fabric, the PSPR and TSP are all significantly decreased. The former decreases from $0.058 \mathrm{~m}^{2} \mathrm{~s}^{-1}$ to $0.013 \mathrm{~m}^{2} \mathrm{~s}^{-1}$, and the latter from $1.516 \mathrm{~m}^{2}$ to $0.391 \mathrm{~m}^{2}$, which decrease by $77.6 \%$

Table 2 Parameters of PAN and FR-PAN by cone calorimetry test

\begin{tabular}{|c|c|c|c|c|c|c|c|c|c|}
\hline Samples & TTI (s) & PHRR (kW m ${ }^{-2}$ ) & Time to PHRR (s) & THR $\left(\mathrm{MJ} \mathrm{m}^{-2}\right)$ & $\operatorname{PSPR}\left(\mathrm{m}^{2} \mathrm{~s}^{-1}\right)$ & $\mathrm{TSP}\left(\mathrm{m}^{2}\right)$ & $\operatorname{aMLR}\left(\mathrm{g} \mathrm{s}^{-1}\right)$ & Residue (wt\%) & FIGRA \\
\hline IIV & $25 \pm 0.2$ & 37 & $45 \pm$ & 7 & & & 05 & & $E$ \\
\hline FR-PAN & $33 \pm 0.2$ & $149.0 \pm 5$ & $55 \pm 0.5$ & $4.5 \pm 0.1$ & $0.01 \pm 0.002$ & $0.4 \pm 0.1$ & $0.01 \pm 0.005$ & $61.6 \pm 2$ & $2.71 \pm 0.3$ \\
\hline
\end{tabular}



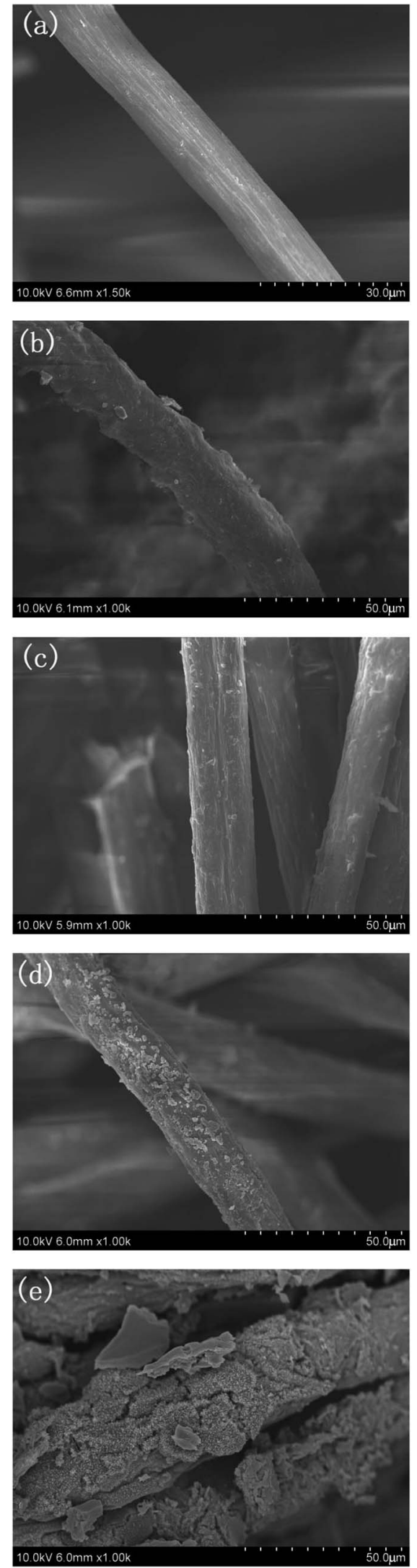

Fig. 7 SEM pictures of different fabrics. (a) PAN; (b) PAN-g-GMA; (c) Am-PAN-g-GMA; (d) FR-PAN; (e) burned FR-PAN. and $74.2 \%$, respectively. It is evident that the smoke production rate and total smoke production of FR-PAN fabric are all decreased greatly, indicating a reduction in fire hazards. The smoke suppression mechanism may be due to the formation of a protective barrier of the resultant compact char residue catalyzed by phosphorus-containing acid species, which thus protects the underlying matrix from degrading into volatile fuel.

\subsection{SEM analysis}

In order to observe the surface morphological features of the PAN, PAN- $g$-GMA, Am-PAN- $g$-GMA and FR-PAN samples, scanning electron microscopy (SEM) was employed to analyse the surface morphology of the samples. As seen in Fig. 7(a), the surface of the pristine fabric is smooth with some grooves. Whereas, PAN- $g$-GMA has rougher surface and some lumps are adhered to the surface, implying the successful grafting of GMA onto the surface of PAN fabric (Fig. 7(b)). For Am-PAN-g-GMA (Fig. 7(c)), the surface morphology is almost the same as that of PAN- $g$-GMA. However, the surface of FR-PAN (Fig. 7(d)) becomes rougher, and a lot of lumps appeared on the surface, suggesting that phosphorylation introduces relative large functional groups onto the surface of the ammoniated PAN- $g$-GMA fabric. As a result, the flame retardancy of the modified PAN fabric increases greatly. The surface of the burned FR-PAN (Fig. 7(e)) becomes coarser and shows dense bark like structure. After phosphorylation, a thin coating will be covered on the surface of PAN fabric and can serve as a protective barrier. When the modified PAN fabric is heated, the coating will be decomposed in advance, resulting in the release of phosphorus-containing acids, which will promote the cyclization of nitrile groups to form much char residue, i.e., it is conductive to effectively inhibit the mass and the heat transfer between the fabrics and the environment during the combustion process. This proves the increased flame retardancy and the amount char residue of the modified PAN fabric as mentioned above.

\subsection{Flame retardant durability of the fabrics}

The LOI values of the PAN and FR-PAN fabric before and after washing are listed in Table 3. The LOI value increases from 17 of the original PAN fabric to 32.4 of the FR-PAN fabric, showing good flame retardancy. Flame retardant durability is an important indicator for evaluating the flame retardancy of materials. Therefore, the flame retardant PAN fabric was washed according to AATCC Tets Method 61-2003 test no. 1A with 0.37 wt $\%$ detergent. With the washing cycles prolonging, the LOI values of the FR-PAN fabric decreases accordingly, and the LOI value is 29.3 after 30 washing cycles, indicating good

Table 3 LOI values of different fabrics before and after washing

\begin{tabular}{llllll}
\hline & \multicolumn{2}{l}{ LOI $(\%)$} & & & \\
\cline { 2 - 6 } Sample & 0 & 5 cycles & 10 cycles & 20 cycles & 30 cycles \\
\hline PAN & 17.0 & - & - & - & - \\
FR-PAN & 32.3 & 32.0 & 31.7 & 31.3 & 29.3
\end{tabular}


washing resistance and excellent flame retardant durability, which attributes to the presence of the covalent bond of phosphorus-containing groups.

\section{Conclusions}

A flame retardant PAN fabric (FR-PAN) was prepared by UVinduced grafted polymerization of GMA onto the PAN fabric followed by ammoniation and phosphorylation. After flame retardant modification, the char residue of the FR-PAN fabric increases greatly and the cyclization temperature decreases compared to the control fabric. The cone calorimeter results show that TTI, time to PHRR and char residue all increase. Whereas, PHRR, THR, TSP, aMLR and PSPR all decrease. In addition, FIGRA decreased by $67.4 \%$, implying good fire hazard suppression. The LOI value of the FR-PAN fabric was 29.3 after 30 washing cycles, indicating good washing resistance and excellent flame retardant durability.

\section{Conflicts of interest}

There are no conflicts to declare.

\section{Acknowledgements}

The authors are grateful to the supports of the National Natural Science Foundation of China (No. 51573134).

\section{References}

1 P. Bajaj, A. K. Agrawal, A. Dhand and N. Kasturia, J. Macromol. Sci., Polym. Rev., 2000, 40(4), 309-337.

2 G. Akovali and G. Gundogan, J. Appl. Polym. Sci., 1990, 41(910), 2011-2019.

3 M. J. Tsafack and J. Levalois-Grutzmacher, Surf. Coat. Technol., 2006, 200(11), 3503-3510.

4 A. Ballistreri, G. Montaudo, C. Puglisi, E. Scamporrino and D. Vitalini, J. Appl. Polym. Sci., 1982, 27(9), 3369-3377.

5 M. E. Hall, J. Zhang and A. R. Horrocks, Fire Mater., 1994, 18(4), 231-241.

6 J. Zhang, A. R. Horrocks and M. E. Hall, Fire Mater., 1994, 18(5), 307-312.

7 P. Wyman, V. Crook, J. Ebdon, B. Hunt and P. Joseph, Polym. Int., 2006, 55(7), 764-771.

8 Y. L. Ren, B. W. Cheng, L. Xu, A. B. Jiang and Y. C. Lu, J. Appl. Polym. Sci., 2010, 115(3), 1489-1494.

9 V. Crook, J. Ebdon, B. Hunt, P. Joseph and P. Wyman, Polym. Degrad. Stab., 2010, 95(12), 2260-2268.

10 D. Y. Zhou, G. Z. Wang, W. S. Li, G. L. Li, C. L. Tan, M. M. Rao and Y. H. Liao, J. Power Sources, 2008, 184(2), 477-480.

11 R. Devasia, C. P. Reghunadhan Nair and K. N. Ninan, Eur. Polym. J., 2002, 38(10), 2003-2010.

12 R. Devasia, C. P. Reghunadhan Nair and K. N. Ninan, Eur. Polym. J., 2003, 39(3), 537-544.
13 Q. Ouyang, L. Cheng, H. J. Wang and K. X. Li, Polym. Degrad. Stab., 2008, 93(8), 1415-1421.

14 Y. L. Ren, Y. T. Gu, Q. Zeng and Y. Zhang, Eur. Polym. J., 2017, 94, 1-10.

15 M. P. Gashti and A. Almasian, Composites, Part B, 2013, 45(1), 282-289.

16 P. Luangtriratana, B. K. Kandola and J. R. Ebdon, Prog. Org. Coat., 2015, 78, 73-82.

17 W. Liu, S. Zhang, X. S. Chen, L. H. Yu, X. J. Zhu and Q. L. Feng, Polym. Degrad. Stab., 2010, 95(9), 1842-1848.

18 C. K. Kundu, B. Yu, C. S. R. Gangireddy, X. W. Mu, B. B. Wang, X. Wang, L. Song and Y. Hu, Ind. Eng. Chem. Res., 2017, 56(6), 1376-1384.

19 C. K. Kundu, X. Wang, Y. B. Hou and Y. Hu, Carbohydr. Polym., 2018, 181, 833-840.

20 A. Kumar, T. Venkatappa Rao, S. Ray Chowdhury and S. V. S. Ramana Reddy, React. Funct. Polym., 2017, 117, 1-9.

21 Y. W. Li, X. W. Zheng, Z. Y. Xia and M. G. Lu, Prog. Org. Coat., 2016, 97, 122-132.

22 Y. M. Deng, J. F. Li, Y. T. Pu, Y. M. Chen, J. L. Zhao and J. N. Tang, React. Funct. Polym., 2016, 103, 92-98.

23 M. Barsbay, O. Guven and Y. Kodama, Radiat. Phys. Chem., 2016, 124, 140-144.

24 X. Chen, Y. Wang, G. L. Dai, J. Peng, J. Q. Li, M. W. Shi and M. L. Zhai, Radiat. Phys. Chem., 2016, 127, 256-263.

25 K. Allmer, A. Hult and B. Ranby, J. Polym. Sci., Part A: Polym. Chem., 1989, 27(5), 1641-1652.

26 J. F. Madrid, G. M. Nuesca and L. V. Abad, Radiat. Phys. Chem., 2013, 85, 182-188.

27 Y. Zhang, Y. L. Ren, X. H. Liu, T. G. Huo and Y. W. Qin, Appl. Surf. Sci., 2018, 428, 395-403.

28 Y. L. Ren, Y. Zhang, Y. T. Gu and Q. Zeng, Prog. Org. Coat., 2017, 112, 225-233.

29 H. H. Sokker, S. M. Badawy, E. M. Zayed, F. A. Nour Eldien and A. M. Farag, J. Hazard. Mater., 2009, 168(1), 137-144.

30 Y. L. Ren, Q. Su and P. Y. Xin, Chem. J. Chin. Univ., 2013, 34(5), 1264-1269.

31 M. Surianarayanan, R. Vijayaraghavan and K. V. Raghavan, J. Polym. Sci., Part A: Polym. Chem., 1998, 36(14), 2503-2512.

32 S. C. Martin, J. J. Liggat and C. E. Snape, Polym. Degrad. Stab., 2001, 74(3), 407-412.

33 Y. L. Ren, T. G. Huo, Y. W. Qin and X. H. Liu, Materials, 2018, 11(4), 483.

34 P. Joseph and S. Tretsiakova-Mcnally, Polym. Degrad. Stab., 2012, 97(12), 2531-2535.

35 A. Q. Ju, S. Y. Guang and H. Y. Xu, Carbon, 2013, 54, 323-335. 36 E. Fitzer, W. Frohs and M. Heine, Carbon, 1986, 24, 387-395. 37 Z. B. Shao, C. Deng, Y. Tan, L. Yu, M. J. Chen, L. Chen and Y. Z. Wang, J. Mater. Chem. A, 2014, 2, 13955.

38 Y. L. Ren, Y. Zhang, J. Y. Zhao, X. L. Wang, Q. Zeng and Y. T. Gu, J. Sol-Gel Sci. Technol., 2017, 82(1), 280-288. 